\title{
Constructing Premaximal Binary Cube-free Words of Any Level
}

\author{
Elena A. Petrova \\ Ural Federal University \\ Ekaterinburg, Russia \\ captain@akado-ural.ru
}

\author{
Arseny M. Shur \\ Ural Federal University \\ Ekaterinburg, Russia \\ arseny.shur@usu.ru
}

\begin{abstract}
We study the structure of the language of binary cube-free words. Namely, we are interested in the cube-free words that cannot be infinitely extended preserving cube-freeness. We show the existence of such words with arbitrarily long finite extensions, both to one side and to both sides.
\end{abstract}

\section{Introduction}

The study of repetition-free words and languages remains quite popular in combinatorics of words: lots of interesting and challenging problems are still open. The most popular repetition-free binary languages are the cube-free language CF and the overlap-free language OF. The language CF is much bigger and has much more complicated structure. For example, the number of overlap-free binary words grows only polynomially with the length [8], while the language of cube-free words has exponential growth [3]. The most accurate bounds for the growth of OF is given in [6] and for the growth of CF in [13]. Further, there is essentially unique nontrivial morphism preserving OF [10], while there are uniform morphisms of any length preserving CF [5]. The sets of two-sided infinite overlap-free and cube-free binary words also have quite different structure, see [12].

Any repetition-free language can be viewed as a poset with respect to prefix, suffix, or factor order. In case of prefix [suffix] order, the diagram of such a poset is a tree; each node generates a subtree and is a common prefix [respectively, suffix] of its descendants. The following questions arise naturally. Does a given word generate finite or infinite subtree? Are the subtrees generated by two given words isomorphic? Can words generate arbitrarily large finite subtrees? For some power-free languages, the decidability of the first question was proved in [4] as a corollary of interesting structural properties. The third question for ternary square-free words constitutes Problem 1.10.9 of [1]. For all $k$ th power-free languages, it was shown in [2] that the subtree generated by any word has at least one leaf. Note that considering the factor order instead of the prefix or the suffix one, we get a more general acyclic graph instead of a tree, but still can ask the same questions about the structure of this graph. For the language OF, all these questions were answered in [11, 14], but almost nothing is known about the same questions for CF.

In this paper, we answer the third question for the language CF in the affirmative. Namely, we construct cube-free words that generate subtrees of any prescribed depth and then extend this result for the subgraphs of the diagram of factor order.

\section{Preliminaries}

Let us recall necessary notation and definitions. We consider finite and infinite words over the binary alphabet $\Sigma=\{a, b\}$. If $x$ is a letter, then $\bar{x}$ denotes the other letter. By default, "word" means a finite word. 
Words are denoted by uppercase characters (to denote one-sided infinite words, we add the subcsript $\infty$ at the corresponding side). We write $\lambda$ for the empty word, and $|W|$ for the length of the word $W$. The letters of nonempty finite and right-infinite words are numbered from 1; thus, $W=W(1) W(2) \cdots W(|W|)$. The letters of left-infinite words are numbered by all nonnegative integers, starting from the right.

We use standard definitions of factors, prefixes, and suffixes of a word. The factor $W(i) \cdots W(j)$ is written as $W(i \ldots j)$. A positive integer $p \leq|W|$ is a period of a word $W$ if $W(i)=W(i+p)$ for all $i \in\{1, \ldots,|W|-p\}$. The minimal period of $W$ is denoted by $\operatorname{per}(W)$. The exponent of a word is the ratio between its length and its minimal period: $\exp (W)=|W| / \operatorname{per}(W)$. Words of exponent 2 and 3 are called squares and cubes, respectively. The local exponent of a word is the number $\operatorname{lexp}(W)=$ $\sup \{\exp (V) \mid V$ is a factor of $W\}$. Periodic words possess the interaction property expressed by the textbook Fine and Wilf theorem: if a word $U$ has periods $p$ and $q$, and $|U| \geq p+q-\operatorname{gcd}(p, q)$, then $U$ has the period $\operatorname{gcd}(p, q)$.

A word $W$ is $\beta$-free $\left[\beta^{+}\right.$-free] if $\operatorname{lexp}(W)<\beta$ [respectively, $\left.\operatorname{lexp}(W) \leq \beta\right]$. The 3 -free words are called cube-free, and the $2^{+}$-free words are overlap-free. The language of all cube-free [overlap-free] words over $\Sigma$ is denoted by CF [respectively, OF]. A morphism $f: \Sigma^{+} \rightarrow \Sigma^{+}$avoids an exponent $\beta$ if the condition $\operatorname{lexp}(U)<\beta$ implies $\operatorname{lexp}(f(U))<\beta$ for any word $U$. The following theorem allowes one to check cube-freeness of a morphism over the binary alphabet.

Theorem 1 ([9]). A morphism $f: \Sigma^{+} \rightarrow \Sigma^{+}$is cube-free if and only if the word $f$ (aabbababbabbaabaababaabb) is cube-free.

The Thue-Morse morphism $\theta$ is defined over $\Sigma^{+}$by the rules $\theta(a)=a b, \theta(b)=b a$. The words

$$
T_{n}^{a}=\theta^{n}(a), T_{n}^{b}=\theta^{n}(b)(n \geq 0)
$$

are called Thue-Morse blocks or simply $n$-blocks. From the definition it follows that $T_{n+1}^{x}=T_{n}^{x} T_{n}^{\bar{x}}$. Hence, the sequences $\left\{T_{n}^{a}\right\}$ and $\left\{T_{n}^{b}\right\}$ have "limits", which are right-infinite Thue-Morse words $T_{\infty}^{a}$ and $T_{\infty}^{b}$, respectively. We also consider the reversal ${ }_{\infty}^{a} T$ of $T_{\infty}^{a}$. The factors of Thue-Morse words are ThueMorse factors; the set of all these factors is denoted by TM. Note that any word in TM can be written as $W=x Q_{1} \cdots Q_{n} y$, where $x, y \in \Sigma \cup\{\lambda\}, Q_{1}, \ldots, Q_{n} \in\{b a, a b\}$. It is known since Thue [15] that TM $\subset$ OF.

Let $L \subset \Sigma^{*}$ and $W \in L$. Any word $U \in \Sigma^{*}$ such that $U W \in L$ is called a left context of $W$ in $L$. The word $W$ is left maximal [left premaximal] if it has no nonempty left contexts [respectively, finitely many left contexts]. The level of the left premaximal word $W$ is the length of its longest left context; thus, left maximal words are of level 0 . The right counterparts of the above notions are defined in a symmetric way. We say that a word is maximal [premaximal] if it is both left and right maximal [respectively, premaximal]. The level of a premaximal word $W$ is the pair $(n, k) \in \mathbb{N}$ such that $n$ and $k$ are the length of the longest left context of $W$ and the length of its longest right context, respectively.

In particular, a word $W \in C F$ is maximal if by adding any of the two letters on the left or on the right we obtain a cube. The word aabaabaa is an example of such a word.

The aim of this paper is to prove the following theorems:

Theorem 2. In CF, there exist left premaximal words of any level $n \in \mathbb{N}_{0}$.

Theorem 3. In CF, there exist premaximal words of any level $(n, k) \in \mathbb{N}_{0}^{2}$.

\section{Construction of premaximal words}

Theorem 2 is proved by exhibiting a series of left premaximal words, containing words of any level. The series is constructed in two steps: 
1. building an auxiliary series $\left\{W_{n}\right\}_{0}^{\infty}$ such that each word $W_{n}$ has, up to one easily handled exception, a unique left context of any length $\leq n$;

2. completing the word $W_{n}$ to a left premaximal word $\bar{W}_{n}$.

If a word $W \in \mathrm{CF}$ has a unique left context of length $n$, say $U$, and two left contexts of length $n+1$, then we say that $U$ is the fixed left context of $W$ (see the picture below).

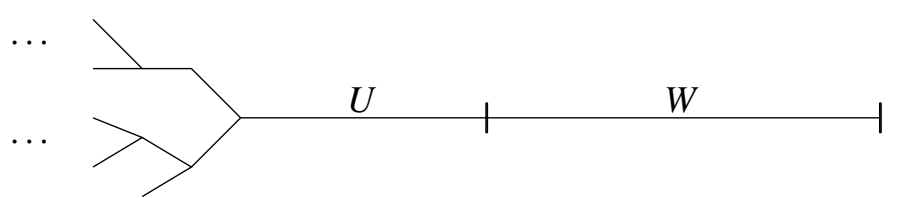

Example 1. Let $W=$ aabaaba. Since $a W=a a a \cdots, a b W=(a b a)^{3}$, but aabbW, babbW $\in \mathrm{CF}$, we see that the fixed left context of the word $W$ equals abb.

Now let us explain step 1 . We build the series $\left\{W_{n}\right\}_{0}^{\infty}$ inductively, one word per iteration, in a way that the fixed left context $X_{n}$ of the word $W_{n}$ is of length $\geq n$ (we will discuss the mentioned exception at the moment of its appearance). We put $W_{0}=a a b a a b a$ and note that the left-infinite word

$$
{ }_{\infty}^{a} T a b a a b a=\cdots a b b a b a a b b a a b a b b W_{0}
$$

is cube-free. So, we require that each word $W_{n}$ satisfies the following properties:

(W1) $W_{n}$ starts with $W_{0}$;

(W2) any word ${ }_{\infty}^{a} T(k \ldots 1)$ is a left context of $W_{n}$;

(W3) some word ${ }_{\infty}^{a} T(k \ldots 1)$ with $k \geq n$ is the fixed left context of $W_{n}$, denoted by $X_{n}$;

(W4) if $\left|X_{n}\right|>n$, then $W_{n+1}=W_{n}$ (trivial iterations).

The basic idea for obtaining $W_{n+1}$ from $W_{n}$ at nontrivial iterations is to let

$$
W_{n+1}=\underbrace{W_{n}} \underbrace{x X_{n} W_{n}} \underbrace{x X_{n} W_{n}},
$$

where $x$ is the letter "prohibited" at the $(n+1)$ th iteration, i.e. $x X_{n}$ certainly is not a left context of $W_{n+1}$. Thus, the fixed left context of $W_{n+1}$ is longer than the one of $W_{n}$ by definition.

Remark 1. An attempt to build the series $\left\{W_{n}\right\}_{0}^{\infty}$ directly by (1) fails because cubes will occur at the border of some words $W_{n}$ and $x X_{n}$. For instance, let us construct the word $W_{4}$. We have $W_{3}=W_{0}$ in view of (W4) and Example $\square X_{3}=a b b$, and the context aabb should be forbidden in view of (W2), because ${ }_{\infty}^{a} T(4 \ldots 1)=b a b b$. So, $x=a$ and the word $W_{3} x X_{3}$ has the factor aaa.

A way out from this situation is the following idea: we insert a special "buffer" word after each of three occurrences of $W_{n}$ in (1). This insertion allows us to avoid local cubes at the border. Below we use the following notation:

- $P_{n}^{\prime}=x X_{n}, P_{n}=\bar{x} X_{n}$, where $x$ is the letter, prohibited at the $(n+1)$ th iteration; thus, $P_{n} \in \mathrm{TM}$;

- $S_{n}$ is the word inserted after $W_{n}$ at the $(n+1)$ th iteration;

- $S_{n}^{\prime}=S_{0} S_{1} \cdots S_{n}$ is the factor of $W_{n+1}$ between $W_{0}$ and the nearest occurrence of $P_{n}^{\prime}$;

- $W_{n}^{\prime}=P_{n}^{\prime} W_{n} S_{n}$. 
In these terms, we have the following expressions for $W_{n+1}$ for any nontrivial iteration:

$$
\begin{aligned}
& W_{n+1}=\underbrace{W_{n} S_{n}} \underbrace{x X_{n} W_{n} S_{n}} \underbrace{W_{n} S_{n}} \underbrace{P_{n}^{\prime} W_{n} S_{n}} \underbrace{P_{n}^{\prime} W_{n} W_{n} S_{n}}
\end{aligned}
$$

The structure of the word $W_{n+1}$ imposes the following restrictions on the words $S_{n}$ and $S_{n+1}$ :

(S1) Since the word $X_{n+1} W_{n+1} S_{n+1}$ is a factor of $W_{n+2}, X_{n+1}$ ends with $X_{n}$, and $X_{n} W_{n+1} x=\left(X_{n} W_{n} S_{n} x\right)^{3}$ by (2a), the word $S_{n+1}$ must start with $\bar{x}$, which is the first letter of $P_{n}$;

(S2) Since the word $S_{n} x X_{n}$ is a factor of $W_{n+1}$, if $X_{n}$ starts with $x[\bar{x} x \bar{x} x]$, then $S_{n}$ ends with $\bar{x}$ [respectively, $x$ ]. (Recall that $X_{n} \in \mathrm{TM}$ is an overlap-free word, whence any other prefix of $X_{n}$ does not restrict the last letter of $S_{n}$.)

Thus, our first goal is to find the words $S_{n}$ satisfying (S1) and (S2) such that all words $S_{n}^{\prime}$ are cube-free. In other words, we have to construct a cube-free right-infinite word $S_{\infty}^{\prime}=S_{0} S_{1} \cdots S_{n} \cdots$. The following lemma is easy.

Lemma 1. The letters ${ }_{\infty}^{a} T(n)$ and ${ }_{\infty}^{a} T(n-1)$ coincide if and only if $n=m \cdot 2^{k}$ for some odd integers $m$ and $k$.

Remark 2. If the only left context of length $n$ of the word $W_{n}$ begins with $x x$, then $\left|X_{n}\right|>n$, because the letter before $x x$ is also fixed. Thus, by (W4) we have $W_{n+1}=W_{n}$ (and then $S_{n}=\lambda$ ) for all values of $n$ mentioned in Lemma 1$]$ For all other values of $n(n>3)$, the iterations will be nontrivial.

While constructing the word $S_{\infty}^{\prime}$ we follow the next four rules:

1. For all nontrivial iterations, $S_{n} \in\left\{T_{2}^{x}, T_{2}^{x} T_{2}^{x}, T_{4}^{x}, T_{2}^{x} T_{2}^{\bar{x}} T_{1}^{x}, T_{1}^{x}, T_{1}^{x} T_{2}^{\bar{x}} \mid x \in \Sigma\right\}$; hence, $S_{n} \in \mathrm{TM}$.

2. Whenever possible, we choose $S_{n}$ to be a 2-block or a product of 2-blocks.

3. Otherwise, if $S_{n}$ ends with the block $T_{1}^{x}$, we put $S_{n+1}=T_{1}^{\bar{x}}$ or $S_{n+1}=T_{1}^{\bar{x}} T_{2}^{x}$ (or the same possibilities for $S_{n+2}$ if $S_{n+1}=\lambda$ ).

4. If $S_{n} \neq \lambda$ and there is no restriction (S2) on the last letter of $S_{n}$, we add this restriction artificially. Namely, we fix the last letter of $S_{n}$ to be $\bar{x}$ if $S_{n-1}$ ends with $x$ (or if $S_{n-2}$ ends with $x$ while $\left.S_{n-1}=\lambda\right)$.

Taking rules $1-4$ into account, we can prove, by case examination, the following lemma about the first and the last letters of the words $S_{n}$.

Lemma 2. (1) If $S_{n}$ ends with $x$, then either $S_{n+1}$ ends with $\bar{x}$, or $S_{n+1}=\lambda$ and $S_{n+2}$ ends with $\bar{x}$.

(2) The first letter of a nonempty word $S_{n}$ coincides with the last one for all n, except for the cases when $P_{n}=x \bar{x} x \bar{x} \cdots$ or $P_{n}=x x \bar{x} x \cdots$.

The construction of the word $S_{\infty}^{\prime}$, the correctness of which we will prove, is given by Table 1 . According to this table, rule 3 applies to $S_{n}$ if and only if $P_{n}$ starts with $x \bar{x} x \bar{x}$. Hence if the word $P_{n}$ has such a prefix, then $P_{n-1}$ (or $P_{n-2}$ if the $(n-1)$ th iteration is trivial) has no such prefix; as a result, the word $S_{n-1}$ (respectively, $S_{n-2}$ ) ends with a 2-block.

Now consider the case $P_{n}=x \bar{x} x \bar{x} \cdots$ in more details. Without loss of generality, let $P_{n}$ start with $b$. Then $P_{n}=$ babaab $\cdots$. Since $P_{n}^{\prime}=a a b a a b \ldots$, the word $S_{n}$ cannot end with $a$ or with $b a a b$; thus, it cannot end with a 2-block and we should use rule 3 . 
Table 1: the suffixes $S_{n}$ for 32 successive iterations starting from some number $k$ divisible by 32 . The righthand [lefthand] part of the table applies if the current letter of $T_{\infty}^{b}$ is equal [resp., not equal] to the previous one. Trivial iterations are omitted.

\begin{tabular}{|l|l|l|l|}
\hline $\begin{array}{l}\text { Iteration no. } \\
(n)\end{array}$ & $\begin{array}{l}\text { Prohibitions } \\
\text { Start }\end{array}$ & End & $S_{n-1}$ \\
\hline$k$ & $\bar{x}$ & $\bar{x}$ & $T_{2}^{x}$ \\
\hline$k+1$ & & & \\
\hline$k+2$ & $x$ & $x$ & $T_{2}^{\bar{x}} T_{2}^{\bar{x}}$ \\
\hline$k+4$ & $\bar{x}$ & $\bar{x}$ & $T_{2}^{x}$ \\
\hline$k+5$ & $\bar{x}$ & $x, T_{2}^{\bar{x}}$ & $T_{2}^{x} T_{2}^{\bar{x}} T_{1}^{x}$ \\
\hline$k+6$ & $x$ & $\bar{x}$ & $T_{1}^{\bar{x}}$ \\
\hline$k+8$ & $x$ & $x$ & $T_{2}^{\bar{x}}$ \\
\hline$k+10$ & $\bar{x}$ & $\bar{x}$ & $T_{2}^{x} T_{2}^{x}$ \\
\hline$k+12$ & $x$ & $x$ & $T_{2}^{\bar{x}}$ \\
\hline$k+13$ & $x$ & $\bar{x}, T_{2}^{x}$ & $T_{2}^{\bar{x}} T_{2}^{x} T_{1}^{\bar{x}}$ \\
\hline$k+14$ & $\bar{x}$ & $x$ & $T_{1}^{x}$ \\
\hline$k+16$ & $\bar{x}$ & $\bar{x}$ & $T_{2}^{x}$ \\
\hline$k+17$ & $\bar{x}$ & $x$ & $T_{1}^{x}$ \\
\hline$k+18$ & $x x \bar{x}$ & $\bar{x}$ & $T_{1}^{\bar{x}}$ \\
\hline$k+20$ & $\overline{x x} x$ & $x$ & $T_{2}^{\bar{x}}$ \\
\hline$k+21$ & $x$ & $\bar{x}$ & $T_{1}^{\bar{x}}$ \\
\hline$k+22$ & $\overline{x x} x$ & $x$ & $T_{1}^{x}$ \\
\hline$k+24$ & $x x \bar{x}$ & $\bar{x}$ & $T_{2}^{x}$ \\
\hline$k+26$ & $x$ & $x$ & $T_{2}^{\bar{x}}$ \\
\hline$k+28$ & $\bar{x}$ & $\bar{x}$ & $T_{4}^{x}$ \\
\hline$k+29$ & $\bar{x}$ & $x, T_{2}^{\bar{x}}$ & $T_{2}^{x} T_{2}^{\bar{x}} T_{1}^{x}$ \\
\hline$k+30$ & $x$ & $\bar{x}$ & $T_{1}^{\bar{x}}\left(T_{1}^{\bar{x}} T_{2}^{x}\right)$ \\
\hline
\end{tabular}

\begin{tabular}{|l|l|l|l|}
\hline $\begin{array}{l}\text { Iteration no. } \\
(n)\end{array}$ & $\begin{array}{l}\text { Prohibitions } \\
\text { Start }\end{array}$ & End & $S_{n-1}$ \\
\hline$k$ & $x$ & $x$ & $T_{2}^{\bar{x}}$ \\
\hline$k+1$ & $x$ & $\bar{x}$ & $T_{1}^{\bar{x}}$ \\
\hline$k+2$ & $\overline{x x} x$ & $x$ & $T_{1}^{x}$ \\
\hline$k+4$ & $x x \bar{x}$ & $\bar{x}$ & $T_{2}^{x}$ \\
\hline$k+5$ & $\bar{x}$ & $x$ & $T_{1}^{x}$ \\
\hline$k+6$ & $x x \bar{x}$ & $\bar{x}$ & $T_{1}^{\bar{x}}$ \\
\hline$k+8$ & $\overline{x x} x$ & $x$ & $T_{2}^{\bar{x}}$ \\
\hline$k+10$ & $\bar{x}$ & $\bar{x}$ & $T_{2}^{x}$ \\
\hline$k+12$ & $x$ & $x$ & $T_{4}^{\bar{x}}$ \\
\hline$k+13$ & $x$ & $\bar{x}, T_{2}^{x}$ & $T_{2}^{\bar{x}} T_{2}^{x} T_{1}^{\bar{x}}$ \\
\hline$k+14$ & $\bar{x}$ & $x$ & $T_{1}^{x}$ \\
\hline$k+16$ & $\bar{x}$ & $\bar{x}$ & $T_{2}^{x}$ \\
\hline$k+17$ & $\bar{x}$ & $x$ & $T_{1}^{x}$ \\
\hline$k+18$ & $x x \bar{x}$ & $\bar{x}$ & $T_{1}^{\bar{x}}$ \\
\hline$k+20$ & $\overline{x x} x$ & $x$ & $T_{2}^{\bar{x}}$ \\
\hline$k+21$ & $x$ & $\bar{x}$ & $T_{1}^{\bar{x}}$ \\
\hline$k+22$ & $\overline{x x} x$ & $x$ & $T_{1}^{x}$ \\
\hline$k+24$ & $x x \bar{x}$ & $\bar{x}$ & $T_{2}^{x}$ \\
\hline$k+26$ & $x$ & $x$ & $T_{2}^{\bar{x}}$ \\
\hline$k+28$ & $\bar{x}$ & $\bar{x}$ & $T_{4}^{x}$ \\
\hline$k+29$ & $\bar{x}$ & $x, T_{2}^{\bar{x}}$ & $T_{2}^{x} T_{2}^{\bar{x}} T_{1}^{x}$ \\
\hline$k+30$ & $x$ & $\bar{x}$ & $T_{1}^{\bar{x}}$ \\
\hline & & & \\
\hline
\end{tabular}

Since $P_{n}$ is a factor of ${ }_{\infty}^{a} T$ while ${ }_{\infty}^{a} T$ is an infinite product of the blocks $T_{2}^{a}=a b b a$ and $T_{2}^{b}=b a a b$, one of the blocks $T_{2}^{a}$ ends in the second position of $P_{n}$. First consider the following occurrence of $P_{n}$ in ${ }_{\infty}^{a} T$ :

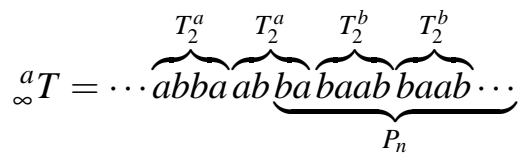

Since $P_{n-1}^{\prime}=b b a a b \cdots$, the word $S_{n-1}$ ends with $a b b a$. Therefore, we cannot put $S_{n}=a b$ (otherwise $S_{n}$ will have the suffix $b a a b$ ). Further, $P_{n-1}$ starts with $a b a a b$, whence the first letter of $S_{n}$ is $a$ by (S1). Hence, according to rule 1 , the only possibility for $S_{n}$ is $T_{2}^{a} T_{2}^{b} T_{1}^{a}=a b b a b a a b a b$. It is easy to see that $S_{n+1}=b a$ satisfies both (S1) and (S2). 
If the last embraced 2-block of (3) is $T_{2}^{a}$, not $T_{2}^{b}$, then we have, up to renaming the letters, the same case as below:

$$
{ }_{\infty}^{a} T=\cdots \overbrace{b a a b}^{T_{2}^{b}} \overbrace{a b b a}^{T_{2}^{a}} \underbrace{T_{b a a b}^{T_{2}^{b}} \cdots}_{P_{n}}
$$

We assign, as above, $S_{n}=T_{2}^{a} T_{2}^{b} T_{1}^{a}$ and $S_{n+1}=T_{1}^{b}$. The problem appears on the $(n+5)$ th iteration, because

$$
P_{n+4}^{\prime}=\underbrace{b} \underbrace{b a b} \underbrace{b a b} a a b \cdots
$$

i.e., $S_{n+4}$ cannot end with $b a$ or $a b$. Here we have an exclusion from the general method. We use the following trick. At the next three iterations $((n+5)$ th to $(n+7)$ th, the last of them being trivial) we have to add the prefix baa to the fixed context. We will do this prohibiting 3-letter contexts instead of single letters. The word $P_{n+3}=b a b b a b a \cdots$ has three left contexts of length 3: $a a b, b a a$, and $b b a$. We will prohibit $b b a$ on the $(n+5)$ th iteration and $a a b$ on the $(n+6)$ th one. To do this, we deliberately put $P_{n+4}^{\prime}=$ bbababbabaab $\cdots, P_{n+5}^{\prime}=$ aabbabbabaab $\cdots$. This allows us to choose $S_{n+4}=b a, S_{n+5}=a b$.

Remark 3. The above trick leads to one local violation of the general rule on $X_{n}$. Namely, $\left|X_{n+5}\right|=n+4$ (this word coincides with $\left.X_{n+4}\right)$. The situation is corrected on the next iteration, when we get $\left|X_{n+6}\right|=$ $n+7$ (and the $(n+7)$ th iteration is trivial).

Remark 4. The word $T_{2}^{a} T_{2}^{a} T_{2}^{b} T_{2}^{a} T_{2}^{a}=\theta^{2}$ (aabaa) is not a factor of ${ }_{\infty}^{a} T$. Hence, the factor $T_{2}^{a} T_{2}^{b} T_{2}^{a}$ occurs in ${ }_{\infty}^{a} T$ inside the factor $T_{2}^{b} T_{2}^{a} T_{2}^{b} T_{2}^{a}$ or $T_{2}^{a} T_{2}^{b} T_{2}^{a} T_{2}^{b}$. Each such factor requires two uses of the above trick with 3-letter contexts.

Let us consider the 108-uniform morphism $\psi: \Sigma^{*} \rightarrow \Sigma^{*}$, defined by the rules

$$
\begin{aligned}
& \psi(a)=T_{4}^{a} T_{2}^{a} T_{2}^{b} T_{2}^{a} T_{4}^{b} T_{2}^{b} T_{2}^{a} T_{4}^{b} T_{2}^{b} T_{2}^{a} T_{2}^{b} T_{4}^{a} T_{2}^{a} T_{2}^{b} T_{2}^{a}, \\
& \psi(b)=T_{4}^{b} T_{2}^{b} T_{2}^{a} T_{2}^{b} T_{4}^{a} T_{2}^{a} T_{2}^{b} T_{4}^{a} T_{2}^{a} T_{2}^{b} T_{2}^{a} T_{4}^{b} T_{2}^{b} T_{2}^{a} T_{2}^{b} .
\end{aligned}
$$

Note that the words $\psi(b)$ and $\psi(a)$ coincide up to renaming the letters. A computer check shows that the word $\psi(a a b b a b a b b a b b a a b a a b a b a a b b)$ is cube-free. Hence by Theorem $1 \psi \psi$ is a cube-free morphism and the word $\psi\left(T_{\infty}^{b}\right)$ is cube-free. So we put $S_{\infty}^{\prime}=\psi\left(T_{\infty}^{b}\right)$. The $\psi$-image of one letter equals the product $S_{n-1} S_{n} \cdots S_{n+30}$ for some number $n$ divisible by 32 , see Table 1 The only exception is described below. Thus, such a $\psi$-image corresponds to 32 successive iterations, during which a 5-block is added to the fixed left context $X_{n-1}$ to get $X_{n+31}$.

There are two different factorizations of the $\psi$-image of a letter, depending on the positions of the factors $T_{2}^{b} T_{2}^{a} T_{2}^{b} T_{2}^{a}$ and $T_{2}^{a} T_{2}^{b} T_{2}^{a} T_{2}^{b}$ inside and on the borders of the current 5-block of ${ }_{\infty}^{a} T$. These factorizations are presented in the two parts of Table 1 . The mentioned factors occur in the middle of $(2 k+1)$-blocks for each $k \geq 2$. Thus, these factors occur in the middle of each 5-block, and also at the border of two equal 5-blocks. For the latter case, the factorization of the $\psi$-image of the second of two equal letters is given in the righthand part of Table 1 In the lefthand part of Table 1, there are two possibilities for $S_{n+29}$ : the longer [shorter] one should be used if the next 5-block is equal [respectively, not equal] to the current one. In the first case, $S_{n+29}$ consists of the last two letters of the $\psi$-image of the current letter and first four letters of the $\psi$-image of the next letter. In the second case, $S_{n+29}$ consists exactly of the two last letters of the $\psi$-image.

The first several iterations are special. Namely, for the regularity of general scheme, we artificially put $W_{3}=W_{0} S_{-1} S_{1}$ (the 1 st and the 3rd iterations are trivial by the general condition).

Thus, we defined the words $S_{n}$ and then the words $W_{n}$ for all positive integers $n$. The correctness of the construction is based on the following lemma. 
Lemma 3. The word $X_{n} W_{n}$ is cube-free for all $n \in \mathbb{N}_{0}$.

Proof. We prove by induction that all the words $V_{n}=\left(X_{n} W_{n} S_{n} x_{n}\right)^{3}$, where $x_{n}$ is the letter forbidden on $(n+1)$ th iteration, have no proper factors that are cubes. This fact immediately implies the statement of the lemma. The inductive base $n \leq 4$ can be easily checked by hand or by computer. Let us prove the inductive step.The structure of the word $V_{n}$ is illustrated by the following picture.

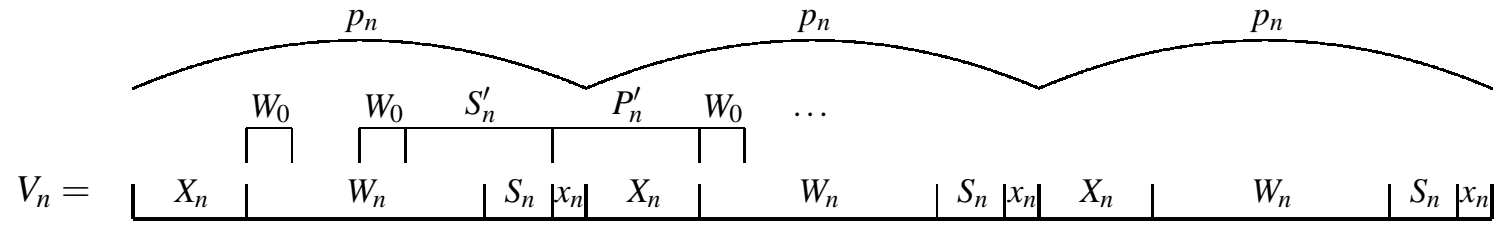

Assume to the contrary that the word $V_{n}, n \geq 5$, contains some cube $U^{3}$. Of course, it is enough to consider the case when the $(n+1)$ th iteration is nontrivial. The factor $U^{3}$ of $V_{n}$ has periods $q=|U|$ and $p_{n}=\left|V_{n}\right| / 3$, but obviously does not satisfy the interaction property. Hence, $\left|U^{3}\right|=3 q \leq q+p_{n}-2$ by the Fine and Wilf theorem, yielding $q \leq p_{n} / 2-1$. On the other hand, by definition of $W_{n}$, the longest proper suffix of the word $X_{n} W_{n}$ coincides with the longest proper prefix of $V_{n-1}$. If $U^{3}$ contains this prefix, then the latter has periods $q$ and $p_{n-1}=\left|V_{n-1}\right| / 3$. Applying the Fine and Wilf theorem again, we get $p_{n-1} \leq q / 2-1$. Excluding $q$ from the two obtained inequalities, we get $p_{n} \geq 4 p_{n-1}+3$. But $p_{n}=\left|V_{n-1}\right|+\left|S_{n}\right|+1 \leq 3 p_{n-1}+17$. Thus, $p_{n-1} \leq 14$. For $n \geq 5$, this is not the case. So, we conclude that $U^{3}$ does not contain the word $X_{n} W_{n}$.

Claim 1. The word $S_{n}^{\prime}$ occurs in $V_{n}$ only three times.

Proof. Recall that $S_{n}^{\prime}$ is a product of 2-blocks (possibly except the last "odd" 1-block), and if $n \geq 5$, then $S_{n}^{\prime}$ begins with a 4-block. Hence, $S_{n}^{\prime}$ has no factor $W_{0}$ and, moreover, cannot begin inside $W_{0}$. Furthermore, it can be checked by hand or by computer that $S_{\infty}^{\prime}$ has no Thue-Morse factors of length $>48$. Now looking at the structure of $S_{n}^{\prime}$ and of $V_{n}$ one can conclude that any "irregular" occurrence of $S_{n}^{\prime}$ in $V_{n}$ should be a prefix of some word $S_{k}^{\prime} P_{k}^{\prime} W_{0}$, where $k<n$. The word $S_{k}^{\prime}$ is a proper prefix of $S_{n}^{\prime}$. The word $P_{k}^{\prime}$ is obtained from a Thue-Morse factor by changing the first letter, and hence never begins with a 2-block. Hence, the only possibility is $k=n-1$, and $S_{n}$ should be the 1-block coinciding with the prefix of $P_{k}^{\prime}$. By Table 1, in all cases when $S_{n}$ is a 1-block, $P_{n-1}^{\prime}$ begins with the square of letter, so this possibility cannot take place.

Claim 2. The word $X_{n} W_{n} S_{n} x_{n}$ is cube-free.

Proof. The word $X_{n} W_{n}$ is a factor of $V_{n-1}$ and hence is cube-free by the inductive assumption. Using again the fact that $S_{n}^{\prime}$ is "almost" a product of 2-blocks, we conclude that $S_{n}^{\prime} x_{n}$ is also cube-free. So, a cube in $X_{n} W_{n} S_{n} x_{n}$, if any, contains inside the suffix $S_{n-1}^{\prime}$ of the word $W_{n}$. This suffix is preceded by $W_{0}=a a b a a b a$; the latter word breaks all periods of $S_{n-1}^{\prime}$ and does not produce a cube. Hence, the cube should contain more than one occurrence of the factor $S_{n-1}^{\prime}$. Applying Claim 1 to the words $S_{n-1}^{\prime}$ and $V_{n-1}$, we see that the cube has the period $p_{n-1}=\left(\left|X_{n} W_{n}\right|+1\right) / 3$. But this is impossible by condition (S1). The claim is proved.

Combining Claim 2 with the fact that $U^{3}$ has no factor $X_{n} W_{n}$, we get that $U^{3}$ is contained inside the word $X_{n} W_{n} S_{n} x_{n} X_{n} W_{n}$. Furthermore, if $S_{n}^{\prime}$ is a factor of $U^{3}$, then the middle occurrence of $U$ is inside $S_{n}^{\prime}$ (otherwise, $U^{3}$ contains one more occurrence of $S_{n}^{\prime}$, contradicting Claim 1). In this case, the positions of all factors $a a$ and $b b$ in $U$ have the same parity. But the rightmost occurrence of $U$ in $U^{3}$ contains a suffix 
of $S_{n}^{\prime}$ followed by a prefix of the word $x_{n} X_{n}=P_{n}^{\prime}$. The letter $x_{n}$ breaks this parity of positions, which is impossible. The cases in which all the positions of $a a$ and $b b$ in the rightmost occurrence of $U$ are on the same side of the letter $x_{n}$, can be easily checked by hand. Thus, we obtain that $S_{n}^{\prime}$ is not a factor of $U^{3}$. Thus, $U^{3}$ begins inside the factor $S_{n}^{\prime} x_{n}$.

Where the word $U^{3}$ ends? It is easy to see that the word

$$
X_{n} W_{n}=\bar{x}_{n-1} X_{n-1} W_{n-1} S_{n-1} x_{n-1} X_{n-1} W_{n-1} S_{n-1} x_{n-1} X_{n-1} W_{n-1} S_{n-1}
$$

has the same three occurrences of the factor $S_{n-1}^{\prime}$ as $V_{n-1}$. So, if $U^{3}$ contains $S_{n-1}^{\prime}$, then the middle occurrence of $U$ is inside $S_{n-1}^{\prime}$. But this is impossible because $S_{n-1}^{\prime}$ is a rather short suffix of $W_{n-1}$ and the whole word $X_{n} W_{n}$ is cube-free. Therefore, $U^{3}$ should end inside the prefix $\bar{x}_{n-1} X_{n-1} W_{n-1} S_{n-1}$ of $X_{n} W_{n}$, like in the following picture.

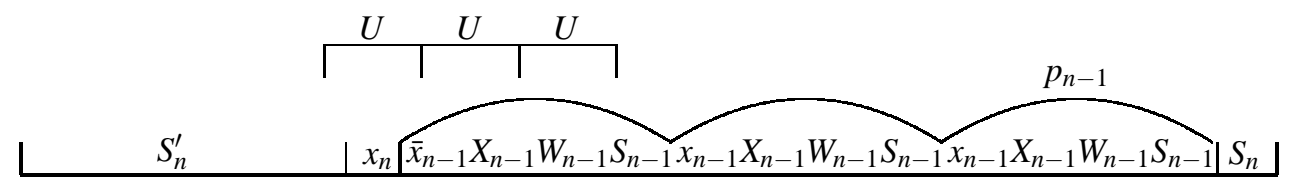

Using the same parity argument as above, we conclude that the word $S_{n}^{\prime} x_{n} X_{n}=S_{n}^{\prime} P_{n}^{\prime}$ is cube-free and, moreover, $U^{3}$ should contain the prefix aabaa of the word $W_{n-1}$. Two cases are to be considered: either aabaa is a factor of $U$ or aabaa occurs in $U^{3}$ only twice, on the borders of consecutive $U$ 's. The second case is impossible, because two closest occurrences of aabaa in $W_{n-1}$ are separated by the factor babaababbaabbabaabaabb which does not contain $P_{n}^{\prime}$ as a suffix. For the first case, we get that some (not the leftmost) occurrence of aabaa in $U^{3}$ is preceded by the concatenation of some suffix of $S_{n}^{\prime}$ and the word $P_{n}^{\prime}$. If this occurrence of aabaa is a prefix of some $W_{0}$, then it is preceded by some $P_{k}^{\prime}, k<n$. But $P_{k}^{\prime}$ is not a suffix of $P_{n}^{\prime}$, a contradiction. The remaining position for this occurrence of aabaa is the border of some words $S_{k}^{\prime}$ and $P_{k}^{\prime}$. But then $S_{k}^{\prime}$ contains the factor which is on the border between $S_{n}^{\prime}$ and $P_{n}^{\prime}$, and the parity argument shows that $S_{k}^{\prime}$ cannot be partitioned into 2-blocks. This final contradiction shows that $U^{3}$ cannot be a factor of $V_{n}$. The lemma is proved.

By construction, the word $X_{n}$ is the fixed left extension of $W_{n}$. Now we consider the second step, that is, the completion of such "almost uniquely" extendable word $W_{n}$ to a premaximal word. The main idea is the same as at the first step. In order to obtain a premaximal word of level $n$, we build the word $W_{n+1}$ in $n+1$ iterations by scheme (2a) and then prohibit the extension of $W_{n+1}$ by the first letter of the word $P_{n}$. We denote the obtained premaximal word of level $n$ by $\bar{W}_{n}$. Then

$$
\bar{W}_{n}=\underbrace{W_{n+1} \bar{S}_{n}} \underbrace{P_{n} W_{n+1} \bar{S}_{n}} \underbrace{P_{n} W_{n+1} \bar{S}_{n}},
$$

where $\bar{S}_{n}$ is a "buffer" inserted similarly to $S_{n}$ in order to avoid cubes at the border of the occurrences of $W_{n+1}$ and $P_{n}$. In contrast to the first step, we do not need to build a cube-free right-infinite word, because the construction (5) is used only once. The form of the word $\bar{S}_{n}$ depends on the last iteration according to Table 1, this dependence is described in Table 2] We choose $\bar{S}_{n}$ to be the left extension of the word $P_{n}$ within ${ }_{\infty}^{a} T$ (recall that $P_{n}={ }_{\infty}^{a} T(n+1 \ldots 1)$ ).

The above idea works without additional gadgets in all cases when $\left|X_{n}\right|=n$. Due to the following obvious remark, it is enough to construct left premaximal words of level $n$ for all $n$ such that $\left|X_{n}\right|=n$; hence, we do not consider constructing the words $\bar{W}_{n}$ for other values of $n$. 
Table 2: the "final" suffixes $\bar{S}_{n}$ for the corresponding iterations from Table 1 The first column contains the number of the last iteration.

\begin{tabular}{|l|l|l|}
\hline $\begin{array}{l}\text { Iteration no. } \\
(n)\end{array}$ & $\begin{array}{l}\text { Prohibitions } \\
(\text { Start })\end{array}$ & $\bar{S}_{n-1}$ \\
\hline$k$ & $\bar{x}$ & $x \bar{x}$ \\
\hline$k+1$ & $x$ & $\bar{x}$ \\
\hline$k+3$ & $x$ & $\lambda$ \\
\hline$k+4$ & $\bar{x}$ & $x \overline{x x} x$ \\
\hline$k+5$ & $\bar{x}$ & $x \bar{x}$ \\
\hline$k+7$ & $x$ & $\bar{x} x$ \\
\hline$k+9$ & $\bar{x}$ & $x$ \\
\hline$k+11$ & $\bar{x}$ & $\lambda$ \\
\hline$k+12$ & $x$ & $\lambda$ \\
\hline$k+13$ & $x$ & $\bar{x}$ \\
\hline$k+15$ & $x$ & $\lambda$ \\
\hline$k+16$ & $x x \bar{x}$ & $x \bar{x}$ \\
\hline$k+18$ & & \\
\hline$k+19$ & $\bar{x}$ & $\lambda$ \\
\hline$k+20$ & $\overline{x x} x$ & $\bar{x} x$ \\
\hline$k+23$ & $\bar{x}$ & $x \bar{x}$ \\
\hline$k+25$ & $x$ & $\bar{x}$ \\
\hline$k+27$ & $x$ & $\lambda$ \\
\hline$k+28$ & $\bar{x}$ & $\lambda$ \\
\hline$k+29$ & $\bar{x}$ & $x$ \\
\hline$k+31$ & & \\
\hline & & $x$ \\
\hline
\end{tabular}

\begin{tabular}{|l|l|l|}
\hline $\begin{array}{l}\text { Iteration no. } \\
(n)\end{array}$ & $\begin{array}{l}\text { Prohibitions } \\
(\text { Start })\end{array}$ & $\bar{S}_{n-1}$ \\
\hline$k$ & $\bar{x}$ & $\lambda$ \\
\hline$k+1$ & & \\
\hline$k+3$ & $\overline{x x} x$ & $\bar{x}$ \\
\hline$k+4$ & $x$ & $\lambda$ \\
\hline$k+5$ & & \\
\hline$k+7$ & $x x \bar{x}$ & $x \bar{x}$ \\
\hline$k+9$ & $x$ & $\bar{x} x$ \\
\hline$k+11$ & $\bar{x}$ & $x$ \\
\hline$k+12$ & $\bar{x}$ & $\lambda$ \\
\hline$k+13$ & $x$ & $\lambda$ \\
\hline$k+15$ & $x$ & $\bar{x}$ \\
\hline$k+16$ & $x$ & $\lambda$ \\
\hline$k+18$ & & \\
\hline$k+19$ & $x x \bar{x}$ & $x$ \\
\hline$k+20$ & $\bar{x}$ & $\lambda$ \\
\hline$k+23$ & $\overline{x x} x$ & $\bar{x} x$ \\
\hline$k+25$ & $\bar{x}$ & $x \bar{x}$ \\
\hline$k+27$ & $x$ & $\bar{x}$ \\
\hline$k+28$ & $x$ & $\lambda$ \\
\hline$k+29$ & $\bar{x}$ & $\lambda$ \\
\hline$k+31$ & $\bar{x}$ & $x \bar{x}$ \\
\hline & & \\
\hline & & \\
\hline$k$ & & \\
\hline & & \\
\hline & & \\
\hline
\end{tabular}

Remark 5. In order to prove the Theorem 2 it is sufficient to show the existence of left premaximal words of level $n$ for infinitely many different values of $n$. Indeed, if a word $W$ is left premaximal of level $n$ and $a_{1} \cdots a_{n} W$ is a left maximal word, then the word $a_{n} W$ is left premaximal of level $n-1$.

Using the facts that $W_{n+1} \in \mathrm{CF}, \bar{S}_{n} P_{n} \in \mathrm{TM}$, and the suffix $S_{n}^{\prime}$ of $W_{n+1}$ has no long Thue-Morse factors (this is the property of any $\psi$-image), we prove the following lemma. The proof resembles the one of Lemma 3.

Lemma 4. The word $X_{n} \bar{W}_{n}$ is cube-free for all $n \in \mathbb{N}_{0}$.

Since the word $P_{n} \bar{W}_{n}$ is a cube by (5) and at the same time $P_{n}=X_{n+1}$ is the fixed left context of $W_{n+1}$, we conclude that $X_{n}$ is the longest left context of the word $\bar{W}_{n}$. Theorem 2 is proved.

Remark 6. For any $n$, the word $\operatorname{rev}\left(\bar{W}_{n}\right)=\bar{W}_{n}\left(\left|\bar{W}_{n}\right|\right) \cdots \bar{W}_{n}(1)$ is right premaximal of level $n$.

Remark 7. Our construction provides an upper bound for the length of the shortest left premaximal word of any given level $n$. The results of [4] suggest that this length is exponential in $n$. Let $l(n)=\left|W_{n}\right|$. For nontrivial iterations, we have $l(n)=3 l(n-1)+O(n)$. It is well known that two successive letters in the Thue-Morse word are equal with probability $1 / 3$. Thus, to obtain $W_{n}$, we make approximately $2 n / 3$ nontrivial iterations. So, $l(n)$ is exponential at base $3^{2 / 3} \approx 2.08$. The same property holds for $\left|\bar{W}_{n}\right|=3 l(n+1)+O(n)$. It is interesting whether this asymptotics is the best possible. 
Sketch of the proof of Theorem 3 Similar to Remark 5 it is enough to build premaximal words of level $\left(n_{i}, n_{i}\right)$ for some infinite sequence $n_{1}<n_{2}<\ldots<n_{i}<\ldots$ of positive integers. We take $n_{i}=32 i+3$ (Table 2 indicates that $\bar{S}_{n_{i}}=\lambda$, which makes the construction easier). The natural idea is to concatenate left premaximal and right premaximal words through some "buffer" word. But we cannot use the words $\bar{W}_{n}$ for this purpose, because all words $X_{n} \bar{W}_{n}$ appear to be right maximal.

So, we modify the last step in constructing left premaximal words as follows. The proof of Lemma 3 implies that the word $X_{n} W_{n} S_{n} \cdots S_{n+l}$ is cube-free for any $l$. So, we put

$$
\widetilde{W}_{n_{i}}=\underbrace{W_{n_{i}+1} S_{n_{i}+1} S_{n_{i}+2}} \underbrace{P_{n_{i}} W_{n_{i}+1} S_{n_{i}+1} S_{n_{i}+2}} \underbrace{P_{n_{i}} W_{n_{i}+1} S_{n_{i}+1} S_{n_{i}+2}} .
$$

By Table 1, $S_{n_{i}+3}=\lambda$ and $S_{n_{i}+4}(1) \neq S_{n_{i}+1}(1)=x$. The proof of the fact that $X_{n_{i}} \widetilde{W}_{n_{i}} \in \mathrm{CF}$ reproduces the proof of Lemma 4 Recall that $S_{n_{i}+1}(1)=P_{n_{i}}(1)$ by (S1), yielding that this letter breaks the period of $W_{n_{i}+1}$ (see (2b) ). On the other hand, the letter $\bar{x}$ breaks the global period of the word $\widetilde{W}_{n_{i}}$. Hence, the condition $X_{n_{i}+1} W_{n_{i}+1} S_{n_{i}+1} \cdots S_{n_{i}+l} \in \mathrm{CF}$ implies $X_{n_{i}} \widetilde{W}_{n_{i}} S_{n_{i}+3} \cdots S_{n_{i}+l} \in \mathrm{CF}$ for any $l$. Thus, $\widetilde{W}_{n_{i}}$ is infinitely extendable to the right, left premaximal word of level $n_{i}$.

Choose an even $m$ such that $\left|X_{n_{i}} \widetilde{W}_{n_{i}}\right|<2^{m-2}$ and consider the word $\widetilde{W}_{n_{i}, n_{i}}=\widetilde{W}_{n} T_{m}^{\bar{x}} \operatorname{rev}\left(\widetilde{W}_{n}\right)$ :

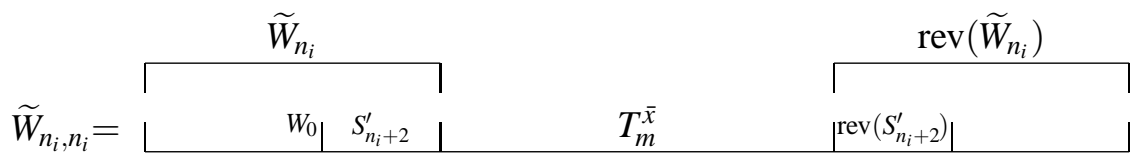

It remains to prove that the word $X_{n_{i}} \widetilde{W}_{n_{i}, n_{i}} \operatorname{rev}\left(X_{n_{i}}\right)$ is cube-free. By the choice of $m$ and overlapfreeness of $T_{m}^{\bar{x}}$, no cube can contain the factor $T_{m}^{\bar{x}}$. So, by symmetry, it is enough to check that the word $U=X_{n_{i}} \widetilde{W}_{n_{i}} T_{m}^{\bar{x}}$ is cube-free. Assume to the contrary that it contains a cube $Y Y Y$. Recall that the word $X_{n_{i}} \widetilde{W}_{n_{i}}$ is cube-free. Since the first letter of $T_{m}^{\bar{x}}$ breaks the period of $X_{n_{i}} \widetilde{W}_{n}$, one has $|Y|<\operatorname{per}\left(\widetilde{W}_{n_{i}}\right)$. Consider the rightmost factor aabaa in $U$; it is inside the factor $W_{0}$ immediately before the suffix $S_{n_{i}+2}^{\prime}$ of $\widetilde{W}_{n}$. If this factor belongs to $Y Y Y$, then $|Y|$ symbols to the left we have another aabaa, followed by $S_{n_{i}+2}^{\prime}$. Then $|Y|=\operatorname{per}\left(\widetilde{W}_{n_{i}}\right)$, a contradiction. Hence, $Y Y Y$ has no factors $a a b a a$, i.e., is a factor of abaaba $S_{n_{i}+2}^{\prime} T_{m}^{\bar{x}}$. One can check that the word $S_{n_{i}+2}^{\prime}$ contains no Thue-Morse factors of length $>48$. The shorter factors can be checked by brute force.

Thus, the word $\widetilde{W}_{n_{i}, n_{i}}$ is premaximal of level $\left(n_{i}, n_{i}\right)$. The theorem is proved.

\section{References}

[1] J.-P. Allouche, J. Shallit (2003): Automatic Sequences: Theory, Applications, Generalizations, Cambridge Univ. Press, doi:10.1017/CB09780511546563.

[2] D. R. Bean, A. Ehrenfeucht, G. McNulty (1979): Avoidable patterns in strings of symbols, Pacific J. Math. 85, 261-294.

[3] F.-J. Brandenburg (1983): Uniformly growing k-th power free homomorphisms, Theor. Comput. Sci. 23, 6982, doi:10.1016/0304-3975 (88) 90009-6.

[4] J. D. Currie (1995): On the structure and extendability of k-power free words, European J. Comb. 16, 111-124, doi:10.1016/0195-6698(95)90051-9.

[5] J. D. Currie, N. Rampersad (2009): There are k-uniform cubefree binary morphisms for all $k \geq 0$, Discrete Appl. Math. 157, 2548-2551, doi:10.1016/j.dam.2009.02.010. Available at http://arxiv.org/abs/0812.4470v1.

[6] R. M. Jungers, V. Y. Protasov, V. D. Blondel (2009): Overlap-free words and spectra of matrices, Theor. Comput. Sci. 410, 3670-3684, doi:10.1016/j.tcs.2009.04.022. Available at http://arxiv.org/abs/0709.1794. 
[7] M. Lothaire (1983): Combinatorics on words, Addison-Wesley, Reading, doi:10.1017/CB09780511566097.

[8] A. Restivo, S. Salemi (2002): Words and Patterns, Proc. 5th Int. Conf. Developments in Language Theory. Springer, Heidelberg, 117-129. (LNCS Vol. 2295), doi:10.1007/3-540-46011-X_9.

[9] G. Richomme, F. Wlazinski (2000): About cube-free morphisms, Proc. STACS'2000. Springer, Berlin, 99109. (LNCS Vol. 1770), doi:10.1007/3-540-46541-3_8.

[10] P. Séébold (1984): Overlap-free sequences, Automata on Infinite Words. Ecole de Printemps d'Informatique Theorique, Le Mont Dore. Springer, Heidelberg, 207-215. (LNCS Vol. 192).

[11] A. M. Shur (1998): Syntactic semigroups of avoidable languages, Siberian Math. J. 39 (1998), 594-610.

[12] A. M. Shur (2000): The structure of the set of cube-free Z-words over a two-letter alphabet, Izv. Math. 64(4), 847-871, doi:10.1070/IM2000v064n04ABEH000301.

[13] A. M. Shur (2009): Two-sided bounds for the growth rates of power-free languages, Proc. 13th Int. Conf. on Developments in Language Theory. Springer, Berlin, 466-477. (LNCS Vol. 5583), doi:10.1007/ 978-3-642-02737-6_38.

[14] A. M. Shur (2011): Deciding context equivalence of binary overlap-free words in linear time, Semigroup Forum. (Submitted)

[15] A. Thue (1912): Über die gegenseitige Lage gleicher Teile gewisser Zeichentreihen, Norske Vid. Selsk. Skr. I, Mat. Nat. Kl. 1. Christiana, 1-67. 